\title{
New Interfacial Shear-Horizontal Waves in Piezoelectric Cubic Crystals
}

\section{A.A. Zakharenko}

International Institute of Zakharenko Waves, Krasnoyarsk, Russia.

E-mail: aazaaz@inbox.ru

Received September $15^{\text {th }}, 2010$; revised October $20^{\text {th }}, 2010$; accepted October $23^{\text {rd }}, 2010$.

\begin{abstract}
This theoretical study reports results on acoustic wave propagation along the interface of two half-spaces representing cubic crystals of both piezoelectric classes $\overline{43} \mathrm{~m}$ and 23 with strong piezoelectric effect. In similar configurations, the interfacial Maerfeld-Tournois waves can propagate along the interface of two transversely-isotropic materials of class $6 \mathrm{~mm}$, in which the shear-horizontal surface acoustic waves $(S H-S A W s)$ called the Bleustein-Gulyaev $(B G)$ waves can also exist. Cubic piezoelectrics cannot support existence of the surface BG-waves, according to the recent report by Gulyaev and Hickernell. Hence, new interfacial SH-waves are studied in this paper concerning unique direction [101] of wave propagation in cubic crystals using different electrical boundary conditions (EBCs) of both metallized and non-metallized interfaces. The new interfacial SH-waves can always propagate along the interface of two identical piezoelectric crystals with opposite polarization. In this case, the calculated velocities for both EBCs coincide with the velocity of the ultrasonic surface Zakharenko wave (USZW) propagating in direction [101] on the metallized surface of a cubic piezoelectrics. It was also found that the new interfacial SH-waves can exist when wave propagation is along the interface of two dissimilar half-spaces, for instance, the piezoelectric cubic crystals $\mathrm{Bi}_{12} \mathrm{SiO}_{20}$ and $\mathrm{Bi}_{12} \mathrm{GeO}_{20}$. Several calculations are also carried out as examples. PACS: 51.40. $+p, 62.65 .+k, 68.35 . G y, 68.35 . I v, 68.60 . B s$, 74.25.Ld.
\end{abstract}

Keywords: New Shear-Horizontal Interfacial Wave, Strong Piezoelectric Effect, Piezoelectric Cubic Crystals, Solutions for "Latent" Waves

\section{Introduction}

The theoretical investigations carried out in 1971 by Maerfeld and Tournois [1] introduced an acoustic shear-horizontal (SH) wave guided by the common interface of two semi-infinite media, if at least one of the media is a piezoelectric material. Maerfeld and Tournois have stated the existence conditions for such waves called the interfacial Maerfeld-Tournois (MT) waves, which can also propagate along the interface of two identical transversely-isotropic crystals of class $6 \mathrm{~mm}$, when the $C$-axes of the crystals are in opposite directions, satisfying the perpendicularity condition of the wave propagation direction to an even-order symmetry axis. This is similar to the other shear-horizontal surface acoustic waves (SH-SAWs) simultaneously discovered by Bleustein [2] and by Gulyaev [3] in the late 1960s, which possess a hybridization between the mechanical displacement $U_{2}$ and electrical potential $\varphi=U_{4}$. These two types of SH-waves, namely the interfacial MT-waves and surface Bleustein-Gulyaev (BG) waves, can only propagate along surfaces of suitable crystal cuts of transversely-isotropic crystals of the hexagonal and tetragonal classes $6 \mathrm{~mm}$ and $4 \mathrm{~mm}$. It is thought that these surface $\mathrm{SH}$-waves have deeper penetration length and lower energy loss than do surface Rayleigh waves [4]. Therefore, they can be easily excited and detected.

The recent work [5] by Gulyaev and Hickernell noted impossibility of SH-SAW existence in piezoelectric cubic crystals. In addition, Ref. [6] discussed that SH-SAWs on electrically open or shorted surfaces of piezoelectric crystals of classes 622 and 422 cannot exist if the propagation direction is perpendicular to six or four-fold axes. However, the surface BG-waves can be found as soon as the transversely-isotropic symmetry decreases from class 622 to 6 or from class 422 to 4 . It is noted that both the surface BG-wave and interfacial electro-acoustic MTwave may be caused by interfacial crack propagation 
between two dissimilar piezoelectric crystals.

The purpose of this report is to introduce theoretical investigations of new interfacial $\mathrm{SH}$-waves in piezoelectric cubic crystals with the strong piezoelectric effect. The Chalcogenide family crystals $\left(\mathrm{Tl}_{3} \mathrm{VS}_{4}\right.$ and $\mathrm{Tl}_{3} \mathrm{TaSe}_{4}$ [7]) belonging to the cubic class $\overline{4} 3 \mathrm{~m}$ and possessing both zero temperature coefficients and strong piezoelectric coupling, are examined. In spite of their very large potential interest, especially for moderate frequency and large bandwidth, such ternary thallium Chalcogenides $\mathrm{Tl}_{3} \mathrm{TaSe}_{4}$ are not commercially available, probably due to their mechanical softness and fabrication difficulties. However, the piezoelectric ceramics of the point group symmetry $23\left(\mathrm{Bi}_{12} \mathrm{SiO}_{20}\right.$ and $\mathrm{Bi}_{12} \mathrm{GeO}_{20}$ [8] and $\mathrm{Bi}_{12} \mathrm{TiO}_{20}$ [9]) can be used in piezoelectronics. Recently, new SH-SAWs [10] called the ultrasonic surface Zakharenko waves (USZWs) were discovered in such cubic piezoelectrics with the strong piezoelectric effect. Note that some piezoelectrics display colossal enhancement of piezoelectricity [11]. It is noted that over several hundred piezoelectric materials, particularly piezoelectric ceramics or composites, are known, for example see Ref. [12], having their intrinsic electromechanical coupling behaviors. They are widely used today for different applications such as filters and sensors, as well as actuators and ultrasonic generators.

Concerning fabrication of a structure consisting of two dissimilar crystals, a process called wafer bonding is utilized in the semiconductor industry, allowing two different materials to be rigidly and permanently bonded along a plane interface, thus producing a composite bi-material [13]. It is noted that several hundred wafer bonding patents are annually deposited [14]. In addition, a similar second process called fusion bonding is used by the polymer industry to bring together two parts of different solid polymers, thus enabling the manufacture of a heterogeneous bi-material with specific properties $[15,16]$. In the first process, wafers of two different crystals are stuck together through Van der Waals forces, after their surface has been mirror-polished. In the second, however, a fusion process followed by a cooling and consolidating period takes place at the interface. It is obvious that it is important to be able to inspect the strength of the bonding, possibly through non-destructive ultrasonic evaluation. However, very few simple numerical investigations exist to compute the speed, etc., of interfacial waves when they exist.

\section{Theory}

Figure 1 introduces a rectangular coordinate system $\left(x_{1}\right.$, $\left.x_{2}, x_{3}\right)$ so that the $x_{1} O x_{3}$ sagittal plane is perpendicular to the even-order symmetry axis of a piezoelectric cubic crystal and the $x_{1}$-axis demonstrates wave propagation in

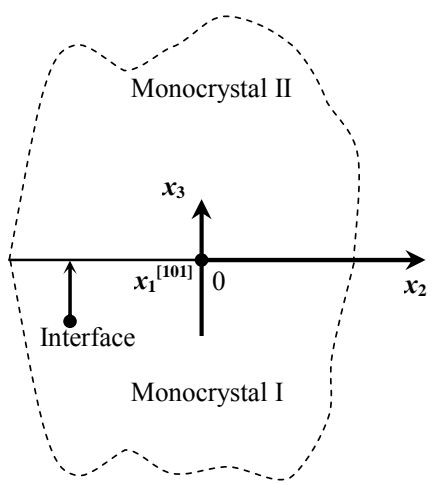

Figure 1. The rectangular coordinate system with the $x_{2}$-axis directed along the interface of two half-spaces where the $x_{1}$-axis is directed perpendicular to the figure plane.

direction [101]. It is necessary to write governing equations of linear piezoelasticity. Constitutive relations are written as follows:

$$
\begin{gathered}
T_{i j}=C_{i j k l}^{E} S_{k l}-e_{i j m} E_{m} \\
D_{m}=e_{m i j} S_{i j}+g_{m n} E_{n}
\end{gathered}
$$

in which $T_{i j}$ and $S_{i j}$ are the stress and strain tensors, respectively; $D_{m}$ and $E_{m}$ are components of the electrical displacement and electrical field $\left(E_{m}=-\partial \varphi / \partial x_{m}\right.$ where $\varphi$ is the electrical potential); the indices $i, j, k, l, m$, and $n$ run from 1 to 3 . According to the Voigt notation, $C_{i j k l}, e_{i j m}$, and $g_{m n}$ can be written as $6 \times 6,3 \times 6$, and $3 \times 3$ matrices standing for the elasticity, piezoelectricity, and dielectricity tensors, respectively. It is assumed that studied materials are free of body forces and inertial effects as well as body electric charge. The equilibrium equations are as follows: $\partial T_{i j} / \partial x_{j}=0$ and $\partial D_{i} / \partial x_{i}=0$.

The equation of motion of an elastic medium is written as follows:

$$
\frac{\partial T_{i k}}{\partial x_{k}}=\rho \frac{\partial^{2} U_{i}}{\partial t^{2}}
$$

where $\rho$ and $U_{i}$ denote the mass density and mechanical displacement components; $t$ is time. Using equations from (1) to (3), the coupled equations of motion for a piezoelectric medium are written in the following forms:

$$
\begin{aligned}
& C_{i j k l} \frac{\partial^{2} U_{l}}{\partial x_{j} \partial x_{k}}+e_{k i j} \frac{\partial^{2} U_{4}}{\partial x_{j} \partial x_{k}}=\rho \frac{\partial^{2} U_{i}}{\partial t^{2}} \\
& e_{i j k} \frac{\partial^{2} U_{k}}{\partial x_{i} \partial x_{j}}-g_{i j} \frac{\partial^{2} U_{4}}{\partial x_{i} \partial x_{j}}=0
\end{aligned}
$$

with $U_{4}=\varphi$. Solutions of the homogeneous partial differential Equation (4) of the second order are found in the following plane wave form: 


$$
U_{i}=U_{i}^{0} \exp \left[j k\left(n_{1} x_{1}+n_{2} x_{2}+n_{3} x_{3}\right)-j \omega t\right]
$$

where the index $i$ runs from 1 to $4 ; U_{i}^{0}$ is an initial amplitude. The imaginary unity is defined as $\mathrm{j}=(-1)^{1 / 2}$ and $\omega$ is the angular frequency. $k$ is the wavenumber in the $k$-space with the following components $\left\{k_{1}, k_{2}, k_{3}\right\}=k\left\{n_{1}\right.$, $\left.n_{2}, n_{3}\right\}$ and $\left\{n_{1}, n_{2}, n_{3}\right\}$ are the directional cosines. $\left\{x_{1}, x_{2}\right.$, $\left.x_{3}\right\}$ are the components of real space.

Leaving only equations for waves with polarization perpendicular to the sagittal plane and non-zero components of the material tensors, the coupled equations of motion in Equation (4) can then be written in the following convenient forms:

$$
\begin{gathered}
C_{44}\left(\frac{\partial^{2} U_{2}}{\partial x_{1}^{2}}+\frac{\partial^{2} U_{2}}{\partial x_{3}^{2}}\right)+e_{16} \frac{\partial^{2} U_{4}}{\partial x_{1}^{2}}+e_{34} \frac{\partial^{2} U_{4}}{\partial x_{3}^{2}}=\rho \frac{\partial^{2} U_{2}}{\partial t^{2}} \\
e_{16} \frac{\partial^{2} U_{2}}{\partial x_{1}^{2}}+e_{34} \frac{\partial^{2} U_{2}}{\partial x_{3}^{2}}-g_{11}\left(\frac{\partial^{2} U_{4}}{\partial x_{1}^{2}}+\frac{\partial^{2} U_{4}}{\partial x_{3}^{2}}\right)=0
\end{gathered}
$$

where the mechanical displacement component $U_{2}$ is directed along the $x_{2}$-axis shown in Figure 1:

$$
U_{2,4}=U_{2,4}^{0} \exp \left[j k\left(n_{1} x_{1}+n_{3} x_{3}-V_{p h} t\right)\right]
$$

In Equation (6), the phase velocity is defined as $v_{p h}=$ $\omega / k$. Equation (5) describes wave propagation in direction [101] with $e_{14}=e_{36}=0$ and the non-zero piezoelectric constants $\left\{e_{16}, e_{34}\right\}$. It is thought that such cuts of cubic crystals can be readily done. Note that all propagation directions perpendicular to direction [010] can exist giving $C_{44}=C_{66}$ and $g_{11}=g_{33}$ concerning piezoelectric cubic crystals.

Using Equations (5) and (6), the tensor form of equations of motion can be written with corresponding GL-components in the Green-Christoffel equations: $G L_{22}=C_{44}\left(1+n_{3}^{2}\right), \quad G L_{24}=G L_{42}=e_{16}+e_{34}+e_{34} n_{3}^{2}$ and $G L_{44}=-g_{11}\left(1+n_{3}^{2}\right)$ where $n_{3}=k_{3} / k$. Therefore, the following system of two homogeneous equations must be resolved:

$$
\left(\begin{array}{cc}
G L_{22}-C_{44}\left(V_{p h} / V_{t 4}\right)^{2} & G L_{24} \\
G L_{42} & G L_{44}
\end{array}\right)\left(\begin{array}{l}
U_{2}^{0} \\
U_{4}^{0}
\end{array}\right)=\left(\begin{array}{l}
0 \\
0
\end{array}\right)
$$

The directional cosines in Equation (7) are defined as follows: $n_{1} \equiv 1, n_{2} \equiv 0$ and $n_{3}=n_{3}$. Setting the determinant of the coefficient matrix in Equation (7) equal to zero, several characteristics can be found such as a suitable phase velocity $V_{p h}$ satisfying boundary conditions discussed in the following section and four polynomial roots $n_{3}{ }^{(p)}\left(V_{p h}\right)$, as well as the functions $U_{2}^{0}\left(V_{p h}\right)$ and $U_{4}^{0}\left(V_{p h}\right)$. It is thought that it is convenient to utilize the functions $U_{2}^{0}\left(V_{p h}\right)$ and $U_{4}^{0}\left(V_{p h}\right)$ in the following forms:
$U_{4}^{0}=G L_{42}$ and $U_{2}^{0}=-G L_{44}$.

Using the piezoelectric constants $e_{16}=-e_{34}$ for direction [101], the following polynomial can be introduced from Equation (7):

$$
\left(1+K_{e}^{2}\right) m_{3}^{2}-B m_{3}+4 K_{e}^{2}=0 \quad \text { with } B=\left(\frac{V_{p h}}{V_{t 4}}\right)+4 K_{e}^{2}(8)
$$

where $m_{3}=1+n_{3}^{2}$. In Equation (8), $K_{e}^{2}$ is the static coefficient of the electromechanical coupling (CEMC): $K_{e}^{2}=e_{16}^{2} /\left(C_{44} g_{11}\right)$. Note that the speed $V_{S H}$ of the bulk SH-wave reads:

$$
V_{S H}=V_{t 4}\left(1+K_{e}^{2}\right)^{1 / 2}
$$

It is obvious that Equation (8) has two roots:

$$
m_{3}^{(1,2)}=\frac{B \pm \sqrt{B^{2}-16 K_{e}^{2}\left(1+K_{e}^{2}\right)}}{2\left(1+K_{e}^{2}\right)}
$$

Hence, four polynomial roots (eigenvalues) of Equation (7) are as follows:

$$
n_{3}^{(1,2,3,4)}= \pm \sqrt{-1+m_{3}^{(1,2)}}
$$

It is noted that the eigenvector $\left\{U_{2}^{0}, U_{4}^{0}\right\}$ in Equation (7) can be obtained for each eigenvalue $n_{3}$.

Note that all complex roots can be calculated when the expression $B^{2}-16 K_{e}^{2}\left(1+K_{e}^{2}\right)$ under square root in Equation (10) is negative. This fulfills for $V_{p h}<V_{K}$ obtained from the following equation $B^{2}-16 K_{e}^{2}\left(1+K_{e}^{2}\right)=$ 0 and defined by the following formula:

$$
V_{K}=\alpha_{K} V_{t 4} \text { with } \alpha_{K}=2 \sqrt{K_{e} \sqrt{1+K_{e}^{2}-K_{e}^{2}}}
$$

It is clearly seen that both the factor $\alpha_{K}$ in Equation (12) and the function $f\left(K_{e}^{2}\right)=\left(1+K_{e}^{2}\right)^{1 / 2}$ in Equation (9) are functions of the CEMC $K_{e}^{2}$. Discussions about behavior of polynomial roots and the function $\alpha_{k}$ can be found in Ref. [10]. However, it is necessary to briefly discuss that only complex polynomial roots can exist for $V_{p h}<V_{K}$ and that for $K_{e}^{2}<K_{0}^{2}\left(K_{0}^{2}=1 / 3\right)$ there are all imaginary roots for $V_{p h}>V_{K}$, but a very large $K_{e}^{2}>K_{0}^{2}$ gives real roots for $V_{p h}>V_{K}$. It is noted that only complex/imaginary roots with negative/positive imaginary parts for medium I/II in Figure 1 are chosen in order to have wave damping towards the each half-space depth from the common interface. Probably, surface waves cannot be found in the cubic crystals with a large $K_{e}^{2}>1 / 3$ in the $V_{p h}$-range: $V_{K}$ $<V_{p h}<V_{S H}$. It is thought that a large $K_{e}^{2}$ can be observed in complex compounds, as well as in simple materials including piezoelectric cubic crystals. For instance, a single ferroelectric tetragonal $(\mathrm{T})$ to paraelectric cubic phase transition is observed in the classic ferroelectric $\mathrm{PbTiO}_{3}$ with increase in temperature or pressure, accord- 
ing to the recent results obtained in Ref. [11].

\section{Boundary Conditions for Interfacial SH-Waves}

There is single boundary at $x_{3}=0$ in Figure 1 representing the interface between two half-spaces, at which suitable requirements can be chosen. These several requirements called boundary conditions must be satisfied for propagation of the interfacial SH-waves, which must damp from the common interface towards depth of each half-space. Using the coordinate system in Figure 1, only eigenvalues with negative imaginary parts must be chosen for the medium with negative values of the $x_{3}$-axis. Also, only eigenvalues with positive imaginary parts must be chosen for the medium with $x_{3}>0$. There are two mechanical boundary condition such as equality of the mechanical displacements $U_{2}\left(U_{2}{ }^{\mathrm{I}}=U_{2}{ }^{\mathrm{II}}\right)$ where

$$
U_{2}^{\mathrm{I}}=\sum_{p=1,2} F^{\mathrm{I}(p)} U_{2}^{\mathrm{I}(p)} \text { and } U_{2}^{\mathrm{II}}=\sum_{p=1,2} F^{\mathrm{II}(p)} U_{2}^{\mathrm{II}(p)}
$$

and equality of the normal components of the stress tensor, $T_{32}^{\mathrm{I}}=T_{32}^{\mathrm{II}}$, where

$$
\left(\begin{array}{cccc}
U_{2}^{\mathrm{I}(1)} & U_{2}^{\mathrm{I}(2)} & -U_{2}^{\mathrm{II}(1)} & -U_{2}^{\mathrm{II}(2)} \\
C_{44}^{\mathrm{I}} k_{3}^{\mathrm{I}(1)} U_{2}^{\mathrm{II}(1)}+ & C_{44}^{\mathrm{I}} k_{3}^{\mathrm{I}(2)} U_{2}^{\mathrm{I}(2)}+ & -C_{44}^{\mathrm{II}} k_{3}^{\mathrm{II}(1)} U_{2}^{\mathrm{II}(1)}- & -C_{44}^{\mathrm{II}} k_{3}^{\mathrm{II}(2)} U_{2}^{\mathrm{II}(2)}- \\
e_{34}^{\mathrm{I}} k_{3}^{\mathrm{I}(1)} U_{4}^{\mathrm{I}(1)} & e_{34}^{\mathrm{I}} k_{3}^{\mathrm{I}(2)} U_{4}^{\mathrm{I}(2)} & e_{34}^{\mathrm{II}} k_{3}^{\mathrm{II}(1)} U_{4}^{\mathrm{II}(1)} & e_{34}^{\mathrm{II}} k_{3}^{\mathrm{II}(2)} U_{4}^{\mathrm{II}(2)} \\
U_{4}^{\mathrm{I}(1)} & U_{4}^{\mathrm{I}(2)} & -U_{4}^{\mathrm{II}(1)} & -U_{4}^{\mathrm{II}(2)} \\
e_{34}^{\mathrm{I}} k_{3}^{\mathrm{I}(1)} U_{2}^{\mathrm{I}(1)}- & e_{34}^{\mathrm{I}} k_{3}^{\mathrm{I}(2)} U_{2}^{\mathrm{I}(2)}- & -e_{34}^{\mathrm{II}} k_{3}^{\mathrm{II}(1)} U_{2}^{\mathrm{II}(1)}+ & -e_{34}^{\mathrm{II}} k_{3}^{\mathrm{II}(2)} U_{2}^{\mathrm{II}(2)}+ \\
g_{33}^{\mathrm{I}} k_{3}^{\mathrm{I}(1)} U_{4}^{\mathrm{II}(1)} & g_{33}^{\mathrm{I}} k_{3}^{\mathrm{I}(2)} U_{4}^{\mathrm{I}(2)} & g_{33}^{\mathrm{II}} k_{3}^{\mathrm{II}(1)} U_{4}^{\mathrm{II}(1)} & g_{33}^{\mathrm{II}} k_{3}^{\mathrm{II}(2)} U_{4}^{\mathrm{II}(2)}
\end{array}\right)\left(\begin{array}{l}
F^{(1)} \\
F^{(2)} \\
F^{(3)} \\
F^{(4)}
\end{array}\right)=\left(\begin{array}{l}
0 \\
0 \\
0 \\
0
\end{array}\right)
$$

Using the mechanical and electrical boundary conditions at $x_{3}=0$ such as $U_{2}^{\mathrm{I}}=U_{2}{ }^{\mathrm{II}}, \sigma_{32}{ }^{\mathrm{I}}=\sigma_{32}{ }^{\mathrm{II}}, \phi^{\mathrm{I}}=0$ and $\phi^{\mathrm{II}}=0$, the

$$
\left(\begin{array}{cccc}
U_{2}^{\mathrm{I}(1)} & U_{2}^{\mathrm{I}(2)} & -U_{2}^{\mathrm{II}(1)} & -U_{2}^{\mathrm{II}(2)} \\
C_{44}^{\mathrm{I}} k_{3}^{\mathrm{I}(1)} U_{2}^{\mathrm{I}(1)}+ & C_{44}^{\mathrm{I}} k_{3}^{\mathrm{I}(2)} U_{2}^{\mathrm{I}(2)}+ & -C_{44}^{\mathrm{II}} k_{3}^{\mathrm{II}(1)} U_{2}^{\mathrm{II}(1)}- & -C_{44}^{\mathrm{II}} k_{3}^{\mathrm{II}(2)} U_{2}^{\mathrm{II}(2)}- \\
e_{34}^{\mathrm{I}} k_{3}^{\mathrm{I}(1)} U_{4}^{\mathrm{I}(1)} & e_{34}^{\mathrm{I}} k_{3}^{\mathrm{I}(2)} U_{4}^{\mathrm{I}(2)} & e_{34}^{\mathrm{II}} k_{3}^{\mathrm{II}(1)} U_{4}^{\mathrm{II}(1)} & e_{34}^{\mathrm{II}} k_{3}^{\mathrm{II}(2)} U_{4}^{\mathrm{II}(2)} \\
U_{4}^{\mathrm{I}(1)} & U_{4}^{\mathrm{I}(2)} & 0 & 0 \\
0 & 0 & U_{4}^{\mathrm{II}(1)} & U_{4}^{\mathrm{II}(2)}
\end{array}\right)\left(\begin{array}{l}
F^{(1)} \\
F^{(2)} \\
F^{(3)} \\
F^{(4)}
\end{array}\right)=\left(\begin{array}{l}
0 \\
0 \\
0 \\
0
\end{array}\right)
$$

BCD4 of the coefficient matrix for the case of metallized interface can be obtained from the following equations: and continuity of the electrical potential $U_{4}=\phi\left(\phi^{\mathrm{I}}=\phi^{\mathrm{II}}\right)$ where

$\phi^{\mathrm{I}}=\sum_{p=1,2} F^{\mathrm{I}(p)} \phi^{\mathrm{I}(p)}$ and $\phi^{\mathrm{II}}=\sum_{p=1,2} F^{\mathrm{II}(p)} \phi^{\mathrm{II}(p)}$

Therefore, the boundary-condition determinant (BCD4) of fourth-order for the interfacial waves can be written using the following four homogeneous equations:

$T_{32}^{\mathrm{I}}=\sum_{p=1,2} F^{\mathrm{I}(p)}\left[C_{44}^{\mathrm{I}} k_{3}^{\mathrm{I}(p)} U_{2}^{\mathrm{I}(p)}+e_{34}^{\mathrm{I}} k_{3}^{\mathrm{I}(p)} U_{4}^{\mathrm{I}(p)}\right]$
$T_{32}^{\mathrm{II}}=\sum_{p=1,2} F^{\mathrm{II}(p)}\left[C_{44}^{\mathrm{II}} k_{3}^{\mathrm{II}(p)} U_{2}^{\mathrm{II}(p)}+e_{34}^{\mathrm{II}} k_{3}^{\mathrm{II}(p)} U_{4}^{\mathrm{II}(p)}\right]$
Note that the superscripts "I" and "II" are for the first and
second media, respectively. There are two electrical
boundary conditions at $x_{3}=0$ : continuity of the normal

$T_{32}^{\mathrm{I}}=\sum_{p=1,2} F^{\mathrm{I}(p)}\left[C_{44}^{\mathrm{I}} k_{3}^{\mathrm{I}(p)} U_{2}^{\mathrm{I}(p)}+e_{34}^{\mathrm{I}} k_{3}^{\mathrm{I}(p)} U_{4}^{\mathrm{I}(p)}\right]$
$T_{32}^{\mathrm{II}}=\sum_{p=1,2} F^{\mathrm{II}(p)}\left[C_{44}^{\mathrm{II}} k_{3}^{\mathrm{II}(p)} U_{2}^{\mathrm{II}(p)}+e_{34}^{\mathrm{II}} k_{3}^{\mathrm{II}(p)} U_{4}^{\mathrm{II}(p)}\right]$
Note that the superscripts "I" and "II" are for the first and
second media, respectively. There are two electrical
boundary conditions at $x_{3}=0:$ continuity of the normal component $D_{3}$ of the electrical displacements, where

$$
\begin{aligned}
& D_{32}^{\mathrm{I}}=\sum_{p=1,2} F^{\mathrm{I}(p)}\left[e_{34}^{\mathrm{I}} k_{3}^{\mathrm{I}(p)} U_{2}^{\mathrm{I}(p)}-g_{33}^{\mathrm{I}} k_{3}^{\mathrm{I}(p)} U_{4}^{\mathrm{I}(p)}\right] \\
& D_{32}^{\mathrm{II}}=\sum_{p=1,2} F^{\mathrm{II}(p)}\left[e_{34}^{\mathrm{II}} k_{3}^{\mathrm{II}(p)} U_{2}^{\mathrm{II}(p)}-g_{33}^{\mathrm{II}} k_{3}^{\mathrm{II}(p)} U_{4}^{\mathrm{II}(p)}\right]
\end{aligned}
$$
(

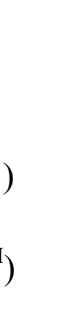


suitable cuts of the transversely-isotropic materials, in which the surface BG-waves can also exist. Note that the velocity of interfacial Maerfeld-Tournois (MT) wave in similar materials with opposite polarization coincides with the velocity $V_{B G m}$ of the surface BG-wave propagating along the metallized surface of transversely-isotropic piezoelectrics, $V_{B G m}=V_{S H}\left[1-\left(K_{e}^{2} /\left(1+K_{e}^{2}\right)\right)^{2}\right]^{1 / 2}$ [2]. This can mean that some similarity occurs between the interfacial MT-waves and surface BG-waves when the transversely-isotropic materials are treated. The velocity $V_{B G}$ of the BG-wave with the non-metallized surface is calculated with the following formula: $V_{B G}=V_{S H}\left\{1-\left(K_{e}^{2} /[(1\right.\right.$ $\left.\left.\left.\left.+K_{e}^{2}\right)\left(1+g_{11} / g_{0}\right)\right]\right)^{2}\right\}^{1 / 2}$. Due to the fact that the surface Bg-waves and the interfacial MT-waves cannot exist in piezoelectric cubic crystals, the existence of new surface $\mathrm{SH}$-waves called the ultrasonic surface Zakharenko waves (USZWs) was studied in the cubic crystals (see Ref. [10]) and the new interfacial SH-wave existence is studied in this paper.

The material constants and USZW characteristics for several cubic crystals with strong piezoelectric effect are listed in Tables 1 and Table 2, respectively. It was found that the velocities of the new interfacial waves with metallized interface are coincide with the $V_{B G m}$. This is also true for the velocities of the interfacial MT-waves and the USZWs on the metallized surface. That illuminates common wave speed characteristics among the different waves. Therefore, they can be calculated with the for mula for $V_{B G m}$ written above. It is noted that all the above mentioned velocities were calculated using different boundary-condition determinants. However, it was found that the velocity of the USZWs on the non-metallized surface cannot be calculated with the formula for $V_{B G}$. For instance, the formula for $V_{B G}$ gives the value of $V_{1} \sim$ $848.6 \mathrm{~m} / \mathrm{s}$ instead of the Table 2 value of $V_{\text {new }} \sim 848.1$ $\mathrm{m} / \mathrm{s}$ for $\mathrm{Tl}_{3} \mathrm{TaSe}_{4}$. The velocity $V_{1}$ is significantly closer to the velocity $v_{S H}$ of bulk waves (see Table 2) than the true velocity $V_{\text {new }}$ obtained numerically. Therefore, it is obvious that the penetration depth of the USZWs in piezoelectric cubic crystals will be smaller than that for the surface BG-waves in the transversely-isotropic monocrystals. This is an advantage of the USZWs. Note that all the wave characteristics were calculated with an accuracy of about $1 \mu \mathrm{m} / \mathrm{s}$. This is useful and allows the distinguishing of $V_{p h}$-solutions when they are close to each other.

Figure 2 shows the phase velocity solutions for the new interfacial waves $\left(V_{i n}\right)$ in the Chalcogenides $\mathrm{Tl}_{3} \mathrm{TaSe}_{4}$ and $\mathrm{Tl}_{3} \mathrm{VS}_{4}$ pertaining to class $\overline{4} 3 \mathrm{~m}, V_{\text {in }}=V_{\text {new, } m}$, using similar materials for the acoustic systems with opposite polarization and both the metallized and non-metallized interfaces, $V_{i n}=V_{i n, m}$. The finding of $V_{i n}$ for the cubic crystals $\mathrm{Bi}_{12} \mathrm{SiO}_{20}$ and $\mathrm{Bi}_{12} \mathrm{GeO}_{20}$ of class 23 is shown in Figure 3 using the same configuration consisting of

Table 1. The material constants: the material density $\rho$, non-zero elastic $C_{44}$, piezoelectric $e_{14}$ and dielectric $g_{11}$ constants for the SH-wave propagation in the piezoelectric cubic crystals $[7,8,9]$ with the strong piezoelectric effect. Note that the dielectric constant of a vacuum is $g_{0}=0.08854\left[10^{-10} \mathrm{~F} / \mathrm{m}\right] ; e_{16}{ }^{[101]}=-e_{14}{ }^{[100]}$ and $e_{34}{ }^{[101]}=e_{14}{ }^{[100]}$. The coefficient of electromechanical coupling (CEMC) $K_{e}^{2}$ in the last column was calculated with the following formula: $K_{e}^{2}=e_{14}{ }^{2} /\left(C_{44} g_{11}\right)$.

\begin{tabular}{lccccc}
\hline \multicolumn{1}{c}{ Material } & Density, $\rho\left[\mathrm{kg} / \mathrm{m}^{3}\right]$ & $C_{44}, 10^{10}\left[\mathrm{~N} / \mathrm{m}^{2}\right]$ & $e_{14}{ }^{[100]}\left[\mathrm{C} / \mathrm{m}^{2}\right]$ & $g_{11} / g_{0}$ & $K_{e}{ }^{2}$ \\
\hline $\mathrm{Tl}_{3} \mathrm{TaSe}_{4}(43 \mathrm{~m})$ & 7280 & 0.410 & 0.320 & 0.1 & 0.2793 \\
$\mathrm{Tl}_{3} \mathrm{VS}_{4}(43 \mathrm{~m})$ & 6140 & 0.470 & 0.550 & 34.8 & 0.2089 \\
$\mathrm{Bi}_{12} \mathrm{TiO}_{20}(23)$ & 11200 & 2.600 & 1.100 & 47.0 & 0.1118 \\
$\mathrm{Bi}_{12} \mathrm{SiO}_{20}(23)$ & 9070 & 2.451 & 1.122 & 41.1 & 0.1412 \\
$\mathrm{Bi}_{12} \mathrm{GaO}_{20}(23)$ & 9300 & 2.600 & 1.100 & 145.7 & 0.0361 \\
$\mathrm{Bi}_{12} \mathrm{GeO}_{20}(23)$ & 9200 & 2.562 & 0.983 & 37.7 & 0.1131 \\
\hline
\end{tabular}

Table 2. The wave characteristics: the velocities $V_{K}, V_{t 4}$, and $V_{S H}($ all in $\mathrm{m} / \mathrm{s})$ as well as the velocities $V_{\text {new }}$ and $V_{\text {new,m }}$ of the new-SH-SAWs (USZWs) on the free and metallized surfaces, respectively, for the piezoelectric cubic crystals with the strong piezoelectric effect when the wave propagation is along direction [101]. The last column lists the non-dimensional values of the CEMC $K^{2}$ calculated with formula (20). Note that one can calculate that $V_{B G}\left(\mathrm{Bi}_{12} \mathrm{SiO}_{20}\right)=1756.096654 \mathrm{~m} / \mathrm{s}$ is larger than $V_{\text {new }}\left(\mathrm{Bi}_{12} \mathrm{SiO}_{20}\right)$ and the same occurs for $V_{B G}\left(\mathrm{Bi}_{12} \mathrm{GeO}_{20}\right)=1760.569112 \mathrm{~m} / \mathrm{s}$.

\begin{tabular}{lccccc}
\hline \multicolumn{1}{c}{ Material } & $V_{K}$ & $V_{t 4}$ & $V_{S H}$ & $V_{\text {new }}$ & $K_{\text {new, } m}$ \\
\hline $\mathrm{Tl}_{3} \mathrm{TaSe}_{4}(\overline{4} 3 \mathrm{~m})$ & 846.9869546 & 750.4577358 & 848.8104580 & 848.125556 & 828.335498 \\
$\mathrm{Tl}_{3} \mathrm{VS}_{4}(\overline{43 \mathrm{~m})}$ & 948.1841318 & 874.9127458 & 961.9607843 & 961.927246 & 9.047 \\
$\mathrm{Bi}_{12} \mathrm{TiO}_{20}(23)$ & 1495.282953 & 1523.62350 & 1606.562692 & 1606.556882 & 1598.414906 \\
$\mathrm{Bi}_{12} \mathrm{SiO}_{20}(23)$ & 1677.148081 & 1643.872053 & 1756.104245 & 1756.089613 & 1742.609095 \\
$\mathrm{Bi}_{12} \mathrm{GaO}_{20}(23)$ & 1326.112526 & 1672.034367 & 1701.927576 & 1701.927574 & 1700.895518 \\
$\mathrm{Bi}_{12} \mathrm{GeO}_{20}(23)$ & 1640.769006 & 1668.766793 & 1760.575183 & 1760.565157 & 1751.469403 \\
\hline
\end{tabular}




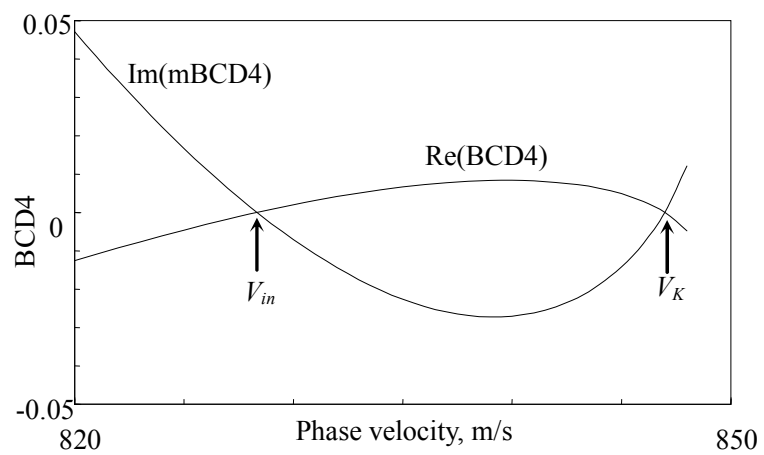

(a)

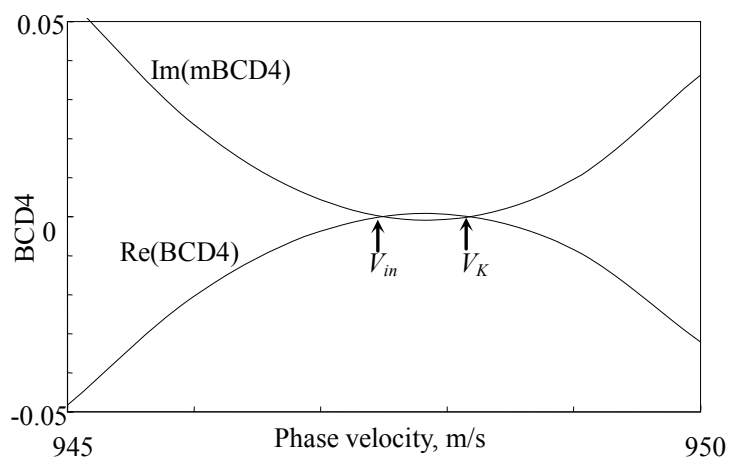

(b)

Figure 2. The dependence of the boundary-condition determinants (BCD4) on the phase velocity $V_{p h}$ for direction [101] of the new interfacial wave $\left(V_{i n}\right)$ propagation in the systems $\mathrm{Tl}_{3} \mathrm{TaSe}_{4}(\mathrm{a})$ and $\mathrm{Tl}_{3} \mathrm{VS}_{4}$ (b) with opposite polarization using the interface metallization (mBCD4) and non-metallized interface (BCD4).

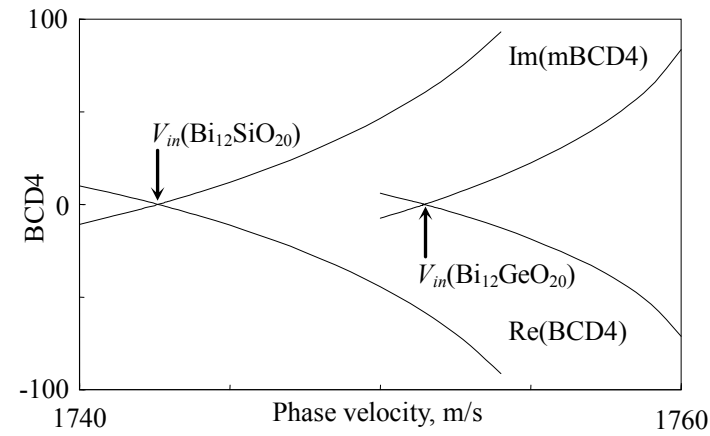

Figure 3. The dependence of the boundary-condition determinants (BCD4) on the phase velocity $V_{p h}$ for direction [101] of the new interfacial wave $\left(V_{i n}\right)$ propagation in the systems $\mathrm{Bi}_{12} \mathrm{SiO}_{20}$ and $\mathrm{Bi}_{12} \mathrm{GeO}_{20}$ with opposite polarization using the interface metallization (mBCD4) and non-metallized interface (BCD4).

similar materials. It was thought that the crystals $\mathrm{Bi}_{12} \mathrm{SiO}_{20}$ and $\mathrm{Bi}_{12} \mathrm{GeO}_{20}$ can be used together to support propagation of the new interfacial waves in dissimilar material, because their bulk $\mathrm{SH}$ velocities $v_{S H}$ are relatively close to each other and also true for $V_{i n}$. The BCD4 behaviors for the dissimilar materials are shown in Figure 4, in which it is clearly seen that there are solutions for the $\mathrm{Bi}_{12} \mathrm{SiO}_{20} / \mathrm{Bi}_{12} \mathrm{GeO}_{20}$ configuration. Using the normal polarization for the crystals $\mathrm{Bi}_{12} \mathrm{SiO}_{20}$ and $\mathrm{Bi}_{12} \mathrm{GeO}_{20}$, only the velocity $V_{i n, m}$ for the metallized interface was found having the value of $V_{i n, m}=$ $1746.926119 \mathrm{~m} / \mathrm{s}$. The $V_{i n, m}$ value is larger than the value of $V_{\text {new, } m}$ for $\mathrm{Bi}_{12} \mathrm{SiO}_{20}$ listed in Table 2 and smaller than the value of $V_{\text {new, } m}$ for $\mathrm{Bi}_{12} \mathrm{GeO}_{20}$. However, the configuration with the opposite-polarized crystals $\mathrm{Bi}_{12} \mathrm{SiO}_{20}$ and $\mathrm{Bi}_{12} \mathrm{GeO}_{20}$ demonstrates existence of both velocities $V_{i n, m}$ and $V_{i n}$ for the metallized and non-metallized interfaces, $V_{i n, m}=1746.926119 \mathrm{~m} / \mathrm{s}$ and $V_{i n}=1747.138149 \mathrm{~m} / \mathrm{s}$. It is clearly seen that the values of $V_{i n, m}$ and $V_{i n}$ are close to each other but distinguishable in the calculations.

In the case of known values of the $V_{i n, m}$ and $V_{i n}$, the existence of the new interfacial waves for both the electrical boundary conditions allows evaluation of the coefficient of electromechanical coupling $\left(K^{2}\right)$ :

$$
K^{2}=2 \frac{V_{i n}-V_{i n, m}}{V_{i n}}
$$

The value of $K^{2}$ calculated with formula (20) is very small for the crystals with the strong piezoelectric effect (see the tables) and is equal to $K^{2} \sim 0.000243$. This is only $\sim 0.0243 \%$ and significantly smaller than $\sim 1 \%$ for $\mathrm{Bi}_{12} \mathrm{GeO}_{20}$ listed in Table 2. It is also noted that the interfacial wave propagation can be studied in suitable two-layer structures consisting of various cubic piezomagnetics $[17,18]$. Note that a study of interfacial wave propagation in non-cubic piezomagnetics was recently introduced in Ref. [19].

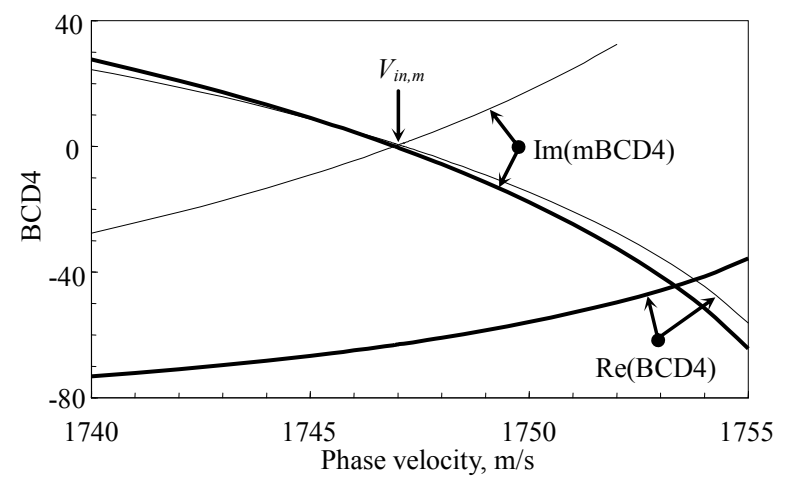

Figure 4. The dependence of the boundary-condition determinants (BCD4) on the phase velocity $V_{p h}$ for direction [101] of the new interfacial wave $\left(V_{i n, m}\right)$ propagation in the $\mathrm{Bi}_{12} \mathrm{SiO}_{20} / \mathrm{Bi}_{12} \mathrm{GeO}_{20}$ systems with normal (bold lines) and opposite polarization (normal lines) using the interface metallization (mBCD4) and non-metallized interface (BCD4). 


\section{Conclusions}

This theoretical report was concerned with the interfacial wave propagation in cubic crystals with strong piezoelectric effect, in which the surface Bleustein-Gulyaev waves cannot exist. This was recently mentioned in Ref. [5] by Gulyaev and Hickernell. It was illuminated that the new interfacial waves can always be found in acoustic systems consisting of two half-spaces representing identical materials with opposite polarization. Also, the new interfacial waves can exist in dissimilar materials solidly coupled at their interface, for instance, in the $\mathrm{Bi}_{12} \mathrm{SiO}_{20} / \mathrm{Bi}_{12} \mathrm{GeO}_{20}$ system, using the electrical boundary conditions of both the metallized and non-metallized interfaces. Note that the theoretically obtained results can be useful to design experiments for measuring interfacial properties by the microwave technologies.

\section{Acknowledgements}

The author is grateful to the Referees for useful notes.

\section{REFERENCES}

[1] C. Maerfeld and P. Tournois, "Pure Shear Elastic Surface Wave Guide by the Interface of Two Semi-Infinite Media," Applied Physics Letters, Vol. 19, No. 4, 1971, pp. 117-118.

[2] J. L. Bleustein, "A New Surface Wave in Piezoelectric Materials," Applied Physics Letters, Vol. 13, No.12, 1968, pp.412-413.

[3] Yu. V. Gulyaev, "Electroacoustic Surface Waves in Solids," Soviet Physics Journal of Experimental and Theoretical Physics Letters, Vol. 9, No.1, 1969, pp. 37-38.

[4] G. A. Maugin, "Elastic Surface Waves with Transverse Horizontal Polarization," Advances in Applied Mechanics, Vol. 23, 1983, pp. 373-434.

[5] Yu. V. Gulyaev and F. S. Hickernell, "Acoustoelectronics: History, Present State, and New Ideas for a New Era," Acoustical Physics Reports, Vol.51, No.1, 2005, pp. 101-110.

[6] G. G. Kessenikh and L. A. Shuvalov, "Transverse Surface Waves in Piezoelectric Crystals of Classes 622 and 422," Ferroelectrics, Vol. 42, No. 1, 1982, pp. 149-152.

[7] J. Henaff, M. Feldmann and M. A. Kirov, "Piezoelectric Crystals for Surface Acoustic Waves (Quartz, $\mathrm{LiNbO}_{3}$,
$\left.\mathrm{LiTaO}_{3}, \mathrm{Tl}_{3} \mathrm{VS}_{4}, \mathrm{Tl}_{3} \mathrm{TaSe}_{4}, \mathrm{AlPO}_{4}, \mathrm{GaAs}\right)$," Ferroelectrics, Vol.42, No.1, 1982, pp. 161-185.

[8] A. A. Zakharenko, "Love Type Waves in Layered Systems Consisting of Two Piezoelectric Cubic Crystals," Journal of Sound and Vibration, Vol. 285, No. 4-5, 2005, pp. 877-886.

[9] V. P. Kamenov, Y. Hu, E. Shamonina, K. H. Ringhofer and V. Ya. Gayvoronsky, "Two-Wave Mixing in (111)-Cut $\mathrm{Bi}_{12} \mathrm{SiO}_{20}$ and $\mathrm{Bi}_{12} \mathrm{TiO}_{20}$ Crystals: Characterization and Comparison with the General Orientation," Physical Review E, Vol. 62, No. 2, 2000, pp. 2863-2870.

[10] A. A. Zakharenko, "New Solutions of Shear Waves in Piezoelectric Cubic Crystals," Journal of Zhejiang University SCIENCE A, Vol. 8, No. 4, 2007, pp. 669-674.

[11] Zh. Wu and R. E. Cohen, "Pressure-Induced Anomalous Phase Transitions and Colossal Enhancement of Piezoelectricity in $\mathrm{PbTiO}_{3}$," Physical Review Letters, Vol. 95, No. 3, 2005, p. 4.

[12] R. C. Pohanka and P. L. Smith, "Recent Advances in Piezoelectric Ceramics", Marcel Dekker, New York, 1988.

[13] U. Gösele and Q. Y. Tong, "Semiconductor Wafer Bonding," Annual Review of Material Science, Vol. 28, 1998, pp. 215-241.

[14] M. Alexe and U. Gösele, "Wafer Bonding," Springer, Berlin, 2003.

[15] C. A. Harper, "Handbook of Plastics, Elastomers and Composites," 3rd Edition, McGraw-Hill, New York, 1996.

[16] C. Ageorges and L. Ye, "Fusion Bonding of Polymer Composites," Springer, Berlin, 2002.

[17] A. A. Zakharenko, "First Evidence of Surface SH-Wave Propagation in Cubic Piezomagnetics," Journal of Electromagnetic Analysis and Applications, Vol. 2, No. 5, 2010, pp. 287-296.

[18] Y. Huang, X.-F. Li and K. Y. Lee, "Interfacial Shear Horizontal (SH) Waves Propagating in a Two-Phase Piezoelectric/Piezomagnetic Structure with an Imperfect Interface," Philosophical Magazine Letters, Vol. 89, No. 2, 2009, pp. 95-103.

[19] A. K. Soh and J. X. Liu, "Interfacial Shear Horizontal Waves in a Piezoelectric-Piezomagnetic Bi-Material," Philosophical Magazine Letters, Vol. 86, No. 1, 2006, pp. 31-35. 